\section{Disfluencia fisiológica en edad preescolar d}

\author{
Physiological disfluency in preschool age
}

Disfluência fisiológica em idlade pré escolar

\section{Karol Stephany Guardia Arce \\ Mayerlin Garrón Prado ${ }^{2}$ Arantxa Valeria Guzmán Rojas ${ }^{3}$}

\section{Resumen}

Introducción. La disfluencia es una alteración involuntaria de la fluidez al hablar. Se caracteriza por la repetición de sonidos, sílabas, palabras o pausas prolongadas. También se refiere a un déficit motor, definiéndolo como una dificultad en la planificación motora, debido a un desfase temporal del mecanismo del habla. Metodología. Se realizó una búsqueda bibliográfica de disfluencias fisiológicas en edad preescolar y su diagnóstico diferencial con disfemia en diversas bases de datos científicas. Desarrollo. Se revisaron 422 títulos, 41 de los cuales cumplieron con nuestros criterios de selección. Se debe tener en cuenta un aspecto importante, que los niños pequeños cuando empiezan a desarrollar el lenguaje presentan este desorden. Discusión y conclusiones. La tartamudez sigue siendo un tema muy investigado, por lo que hemos encontrado una variedad de artículos, aunque muchos de ellos van en direcciones opuestas. Principalmente por su etiología, dado que aún no se conoce la causa exacta, suelen aparecer entre los 3 y los 5 años de edad. Pueden ser muy frecuentes en niños que desarrollan habilidades comunicativas, no en todos los casos. Por lo tanto, rara vez se vuelve grave, que sería del $1 \%$.

Palabras clave: Tartamudeo, Desarrollo Infantil, Trastornos del Desarrollo del Lenguaje, Preescolar. (Fuente: Descriptores en Ciencias de la Salud DeCS).

\section{Abstract}

Introduction. The disfluency is an involuntary disturbance of fluency when speaking. It is characterized by the repetition of sounds, syllables, words or long breaks. It is also refered to a motor deficit, defining it as a difficulty in the motor planning, due to time lag of the speech mechanism. Methodology. A bibliographic search is carried out about physiological disfluencies in preschool age and its differential diagnosis with dysphemia in various scientific databases. Development. They were revised 442 titles, 41 of them met the selection criteria. It must be taken into account an important aspect that the small children when they begin to develop the language, they present this speech
Correspondencia a:

${ }^{1}$ Centro de investigación de fonoaudiología UNITEPC. "CIFU".

Cochabamba - Bolivia.

${ }^{2}$ Licenciado en Fonoaudiología.

Cochabamba - Bolivia

${ }^{3}$ Médico Cirujano. Centro de investigación de Salud Pública UNITEPC "CISPU". Cochabamba - Bolivia. Email de contacto:

karolguardia24@gmail.con mayerlincita019@hotmail. com

aravaleguzman94@gmail. com

Procedencia y arbitraje:

No comisionado, sometido a arbitraje externo

Recibido para publicación 29 de febrero del 2021 Aceptado para publicación: 20 de mayo del 2021

Citar como:

Guardia Arce KS, Garrón Prado M, Guzmán Rojas AV. Disfluencia fisiológica en edad preescolar. Recisa UNITEPC. 8 de junio de 2021;8(1):45-6.

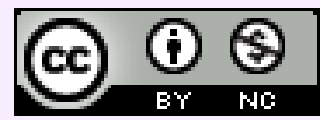

Esta obra está bajo una Licencia Creative Commons Atribución-NoComercial 4.0 Internacional. 
disorder. Discussion and conclusions. The stuttering remains a highly researched topic so that it has been found a variety of articles although many of them go in opposite direcctions. Mainly for its etiology that it is not yet know the exact cause. It usually occurs between 3 and 5 years old. They can be frequent in children that develop communicative skills but not in all cases. Therefore rarely do it get serious that would be from $1 \%$.

Keywords: Stuttering, Child Development, Language Development Disorders, Child, Preschool. (source: Health Sciences Descriptors DeCS)

\section{Resumo}

Introdução: A disfluência é uma alteração involuntária da fluidez ao falar. Caracteriza-se pela repetição de sons, sílabas, palavras ou pausas prolongadas. Também se refere a um déficit motor, definindo-o como uma dificuldade no planejamento motor, devido a um lapso de tempo do mecanismo de fala. Metodologia: Realizou-se uma pesquisa bibliográfica sobre disfluências fisiológicas em idade pré-escolar e seu diagnóstico diferencial, com disfemia, foi realizado em várias bases de dados científicas. Desenvolvimento: Foram revisados 422 títulos, dos quais, 41 atenderam aos critérios de seleção. Um aspecto importante a ser levado em consideração, é que, crianças pequenas quando começam a desenvolver a linguagem, podem apresentam esta desordem. Discussão e conclusões: A Tartamudez continua sendo um tema muito investigado e, portanto, vários artigos foram encontrados, embora muitos deles sigam em direções opostas. Principalmente pela sua etiologia, já que a causa exata ainda não é conhecida, costumam aparecer entre 3 e 5 anos de idade. Eles podem ser muito comuns em crianças que desenvolvem habilidades de comunicação, não em todos os casos. Portanto, raramente se torna grave, seria $1 \%$.

Palavras-chave: Gagueira, Desenvolvimento Infantil, Transtornos do Desenvolvimento da Linguagem, Pré-Escolar. (fonte: Descritores em Ciências da Saúde: DeCS).

\section{Introducción}

La disfluencia o más conocida con el nombre de tartamudez es una alteración involuntaria en la fluidez del habla. Se caracteriza por la repetición de sonidos, sílabas, palabras o las pausas prolongadas al hablar. Asímismo la literatura señala que la tartamudez concierne a un déficit motor, definiéndola como una dificultad en la planeación motora, debido a un desfase temporal del mecanismo de habla $(1,2)$.

Se denomina fisiológica porque generalmente desaparece sola, ya que se la considera como una etapa normal del desarrollo de lenguaje, siendo una señal de alarma la presencia de esfuerzos, gestos o movimientos al hablar (3)aimed at evaluating and intervening in speech disorders (dyslalia, stuttering, diglossia and dysarthria. Se constituye como la forma más frecuente, en torno al $80 \%$ de los casos, que ocurre en niños que están desarrollando las habilidades del lenguaje, generalmente entre los tres años.

Típicamente se inicia de manera gradual, presentándose en pacientes con un desarrollo del lenguaje normal. Surgiendo cuando las habilidades del habla de los niños no alcanzan a cubrir las demandas verbales del mismo, trascurriendo varios meses durante los cuales se producen anomalías de la fluidez verbal episódica desapercibidas, las que llevan a un problema crónico. 
La mayoría de los científicos y profesionales médicos creen que el tartamudeo del desarrollo proviene de las interacciones complejas de diversos factores, que no se asocian a causas ambientales concretos y la timidez no supone un factor de riesgo. Los elementos asociados pueden ser: fisiológicos, psicosociales y lingüísticos (4-6).

La edad preescolar abarca de los 3 a los 5 años que según Jean Piaget comprende el segundo periodo de desarrollo infantil y lo llama preoperacional y es donde el niño adquiriere un acelerado progreso de la expresión.

Comienza usando palabras en forma verdaderamente representacional, las usa para referirse a eventos que no experimenta de modo directo. En esta etapa el infante organiza y estructura el lenguaje interior, el cual trata de expresar correctamente y es aquí frecuentemente cuando aparecen los diversos problemas (7-9).

Aproximadamente un tercio de la población de niños de 2,5 a 5 años pasan por una fase de tartamudeo. En la gran mayoría remitirá sin dejar ningún tipo de huella (cerca de $75 \%$ y el $85 \%$ ), a esta etapa se le denomina tartamudez fisiológica o evolutiva, el resto (entre el 15 al 25\%) mantendrá la disfluencia por un tiempo mayor a los catorce meses. Solo un pequeño porcentaje de este grupo se convertirá en adultos tartamudos

Esto también se presenta con frecuencia familiar y hay más niños que niñas que tartamudean, con una proporción aproximada de 4 a 1. La prevalencia del trastorno se estima en un $1 \%$ en la población general y entre los 3 y los 4 años se encuentra el mayor riesgo para tartamudear (10).

A nivel mundial a través de estudios se conoce que la incidencia es más en los hombres que en mujeres en un porcentaje de $75 \%$ y $80 \%$. Esto debe verse en un sentido evolutivo, ya que hasta los cinco años la proporción es solo de 2 a 3 niños por niñas, en edad escolar es de 4 a 5 y los adultos es de 6 a 11. Estos datos indican el carácter progresivo de la tartamudez y que las personas de sexo femenino sé recuperando mejor (11).

Se realizaron estudios recientes en el municipio cerró en la Ciudad de la Habana en las cuatro áreas de salud, en donde la muestra fueron 312 niños en la entrada del círculo infantil, de los cuales el $0.69 \%$ presentaba tartamudez (12).

Por lo tanto, la tartamudez fisiológica, es frecuente entre la edad preescolar, aunque no se da en todos los casos, frecuentemente suele desaparecer de manera espontánea siempre y cuando se actúe de forma correcta. No obstante, si existen antecedentes familiares, situaciones de bloqueos muy frecuentes, acompañado de comportamientos inusuales, debemos acudir a un logopeda, ayudando así al niño a mejorar el habla para poder obtener una mejor calidad de vida (13).

La disfluencia del desarrollo es una alteración de la fluidez del habla que dificulta la comunicación, afectando frecuentemente a los infantes en la edad preescolar, usualmente suele desaparecer por completo en un tiempo determinado sin ocasionar ningún tipo de secuelas. Pero no en todos los casos suelen ser pasajero ni se da en la totalidad los niños en esta etapa, en muchas ocasiones la tartamudez puede quedarse de por vida, ocasionando en el niño un lenguaje menos fluido. Es aquí donde desarrolla un problema en la expresión oral, presentando limitaciones en la articulación de los sonidos y palabras. Siendo en este periodo que muchos desconocen la información, 
sobre cuando debería de preocuparnos.

Por lo tanto, una inadecuada intervención en el niño influirá en el desarrollo del lenguaje dificultando sus relaciones personales o sociales, al no poder comunicarse de forma adecuada con las demás personas.

La revisión será de beneficio para profesionales que trabajan en el área del lenguaje y expresión, padres de familia y sobre todo para niños en edad preescolar que atraviesan por esta etapa. Al ofrecer información se estará atento a cuando se debería actuar o intervenir en el habla del niño, logrando así una intervención temprana, en donde podrá adquirir habilidades para lograr una comunicación eficaz con mejores resultados.

Se realizará una recopilación bibliográfica sobre las difluencias fisiológicas en edad preescolar y su diagnóstico diferencial con la disfemia.

\section{Metodología}

Se realizó una búsqueda bibliográfica de las difluencias fisiológicas en edad preescolar y su diagnóstico diferencial con las disfemias en diferentes bases de datos científicas.

Los criterios de inclusión fueron artículos en inglés y español publicados, con acceso gratuito al texto completo. Se realiza una primera selección de los estudios relevantes por título de estudio y se excluyeron aquellas que no eran pertinentes por no ajustarse a lo establecido.

\section{Desarrollo}

Desarrollo: Se revisaron 422 títulos, 41 de los cuales cumplieron con nuestros criterios de selección.

Al definir qué es la tartamudez se debe tener en cuenta un aspecto importante, que los niños pequeños cuando empiezan a desarrollar el lenguaje presentan disfluencias fisiológicas. Que son consideradas como una etapa normal del desarrollo, que al quedarse por un periodo prolongado constituyen un problema que puede interferir en el diario vivir del niño (14).

Es un trastorno del lenguaje, específicamente en la fluidez verbal, por eso actualmente se la denomina disfluencia. Se la puede definir como un desorden en el curso fluido de los movimientos del habla que se caracteriza por una serie de alteraciones, repeticiones o prolongaciones de sonidos palabras o frases y bloqueos o pausas inadecuadas en el discurso.

Aunque en la organización de los movimientos del habla las vías neurológicas estén intactas, la coordinación al pronunciar palabras puede producirse en forma deficiente (15).

Según el DSM-V la tartamudez es una alteración en la fluidez y la organización temporal del habla que se manifiesta por uno o más de los siguientes síntomas:

- Repeticiones, por ejemplo, en sonidos palabras o frases como; (b-b-bebe), (pepe-perro), (pero, pero, pero, yo quiero ir).

- Prolongaciones, de sonidos por ejemplo (aaaaaaa, a veces nos quedamos afuera)

- Palabras fragmentadas, que son las pausas en medio de una palabra como, por 
ejemplo: (quiero la mu......ñeca)

- Bloqueos, se refiere a las pausas que impiden al niño hablar, que suele ir acompañado de movimientos o tensión en la cara y el cuerpo en donde intenta realizar un sonido, pero no puede.

- Circunloquios, que consiste en la sustitución de una palabra difícil de pronunciar para el hablante.

- Palabras producidas con exceso de tensión física.

- Repeticiones de palabras monosilábica, como, por ejemplo: (quiero comer pan, pan, pan, pan) Que generalmente inicia durante el desarrollo del lenguaje del niño (16).

\section{Clasificación de las disfluencias:}

- Tónica, se producen cuando al hablar parece entrecortada por bloqueos que afectan a grupos musculares relacionados con la fonoarticulación. Lo que conlleva a un habla explosiva y violenta a causa del gran esfuerzo y aumento del tono muscular que realiza el sujeto para solucionarlo (17).

- Clónica, caracterizada por las repeticiones de sílabas y palabras enteras, frecuentes en consonante que, en vocal, más todavía en oclusivas y que se dan a principio que en medio de palabra.

- Mixta, es el tipo más frecuente, porque resulta difícil encontrar un disfémico puro tónico o clónico, la mayoría combinan los dos síntomas (18).

Las causas de las disfluencias aún no están del todo claras, por lo cual hemos recopilado las teorías que diversos autores ha aportado como causa, lo cierto es que la tartamudez hoy en día se puede considerar multifactorial entre estas tenemos:

Genéticas. - Causas genéticas, se han identificado varios grupos familiares en los que existen sujetos con esta dificultad teniendo progenitores disfémicos. En gemelos monocigóticos si uno sufre tartamudez el otro tiene un $90 \%$ de posibilidades de sufrirlo, mientras que en los dicigóticos es de un $25 \%$. Asimismo, existe entre un $30 \%$ y un $40 \%$ de probabilidad de que el hijo de un progenitor disfémico tenga este trastorno. A pesar de todos estos datos, no hay evidencia de que el problema sea hereditario, aunque sí una cierta predisposición a padecerlo. La disfemia es más frecuente en hombres $(75 \%)$ que mujeres. Además, el número de recuperaciones espontáneas es mayor, un $66 \%$ el sexo femenino, que en el masculino un $46 \%$ (19).

Teoría psicosocial. - Algunos factores relacionados con el mantenimiento del trastorno son la actitud negativa de los interlocutores, la atención a los errores y las correcciones realizadas a los niños, así como un alto nivel de la exigencia por parte de los padres, o escasas habilidades sociales, tendencia al retraimiento o baja autoestima por parte del niño.

Desde el modelo conductual no existe un modelo unificado para explicar la tartamudez, pero se entiende el mantenimiento del problema mediado por experiencias de condicionamiento directo. Dentro de esta perspectiva se estableció un modelo que explica la disfemia como un proceso de condiciones de ansiedad al habla. Antes las disfluencias normales en el comienzo del habla se producirían recriminaciones al niño por parte del entorno. La ansiedad que estas generan facilita la aparición de nuevas alteraciones en el habla, al aumentar la tensión en el sistema fonador (20). 
Teoría psicolingüística. - Sugiere que comienza durante la planeación del habla, esto es, mucho antes que la producción oral. Esta teoría propone que son resultado de un déficit en la estructuración del lenguaje, un sistema fonológico lento desencadena un mayor número de errores y una alta demanda de auto reparaciones que interrumpen el fluido de la comunicación verbal. Es decir que los niños con la alteración tienen un esquema fonológico/articulatorio menos desarrollado u organizado que aquellos con fluidez normal; resultando la tartamudez como un problema en la codificación fonológica $(21,22)$.

\section{Evolución de la disfluencia fisiológica en la etapa preescolar}

Las disfluencias son consideradas una etapa normal del desarrollo, por ello pasa por etapas las cuales identificaremos como:

Primarias. - Aparece hacia los tres años aproximadamente, coincidiendo con la estructuración sintáctica del lenguaje infantil y no en las primeras etapas de la adquisición del mismo, presentando, por tanto, un aspecto evolutivo. Este tipo de tartamudeo inicial desaparece con la edad, de ahí que haya autores que hablen de primaria, fisiológica, de desarrollo o balbucencia, en esta etapa. El niño presentará repeticiones cortas sin esfuerzos y aún no estará consiente de su tartamudez $(23,24)$.

Transicionales. - A medida que el trastorno progresa, las repeticiones se hacen frecuentes y el tartamudeo se hace patente en las palabras o frases importantes. Según el niño va percibiendo sus dificultades verbales, va tomando conciencia de sus problemas (tartamudez transicional) comienzan a ejecutar estrategias más o menos inadecuadas con el fin de no tartamudear (entre ocho y doce años) $(25,26)$.

Secundaria o disfemia. - Ocurre cuando estos intentos de solucionar la tartamudez son inadecuados, aumentan en mayor medida los errores de dicción y, en la magnitud en que el sujeto utiliza estas estrategias, se constituye lo que llamamos un individuo disfémico o tartamudo. Esto le lleva, en la mayoría de los casos, a tener unas determinadas expectativas sobre la aparición del tartamudeo. Al condicionamiento a establecidas situaciones relacionadas con habla, a evitar hablar lo más posible, e incluso, a modificar la estructura del discurso a nivel fonológico y gramatical $(27,28)$.

\section{Diferencia de la disfluencia fisiológica y la disfemia}

Está descrito que el $75 \%$ al $80 \%$ de los niños que presentan disfluencia es entre los 3 años, se recuperan espontáneamente. El otro $20 \%-25 \%$ empiezan a luchar por producir las palabras y terminan tartamudeando. Para estos las disfluencias no concluyen formando parte del desarrollo normal del habla y del lenguaje. Aunque las repeticiones en los infantes pequeños pueden ser consideradas normales, cuando estas comienzan a aumentar en su frecuencia de aparición, no deben ser tomadas como típicas en el hablar diaria del niño. Si este aumento va acompañado de movimientos asociados de la cara o del cuerpo para tratar de decir la palabra, esto se considera un signo de alerta que amerita la evaluación de un terapista especializado (29).

Por ello hemos buscado una tabla comparativa, entre las disfluencias y la disfemia que derivamos de tomar en cuenta al momento de preocuparnos: (30-32)"container-title":"Revista de Logopedia, Foniatría y Audiología","DOI":"10.1016/S0214-4603(00 


\begin{tabular}{|c|c|}
\hline $\begin{array}{l}\text { Disfluencias fisiológicas } \\
\text { Tienen una frecuencia de errores al hablar me- } \\
\text { nor. }\end{array}$ & $\begin{array}{l}\text { Disfemia } \\
\text { Tienen una frecuencia de errores al hablar mayor }\end{array}$ \\
\hline $\begin{array}{l}\text { La repetición entera de la palabra, repite sola- } \\
\text { mente una vez y sin tensión }\end{array}$ & $\begin{array}{l}\text { Repetición de una palabra monosilábica, repetida } \\
\text { dos o más veces, especialmente si el niño muestra } \\
\text { tensión o aumento de tono }\end{array}$ \\
\hline $\begin{array}{l}\text { Repetición de palabra multisilaba (gallina-galli- } \\
\text { na) }\end{array}$ & $\begin{array}{l}\text { Parte de la palabra repetida (co-co-co-co-copa) dos } \\
\text { veces o más. Especialmente si el niño muestra ten- } \\
\text { sión o aumento de tono }\end{array}$ \\
\hline $\begin{array}{l}\text { Un sonido es prolongado brevemente, por me- } \\
\text { nos de un segundo solo rara vez sucede esto }\end{array}$ & $\begin{array}{l}\text { Un sonido es prolongando por } 1 \text { segundo o más si } \\
\text { hay pitch aumentando durante la prolongación }\end{array}$ \\
\hline $\begin{array}{l}\text { No hay sonidos completamente bloqueados, } \\
\text { con evidente paralización del habla. }\end{array}$ & $\begin{array}{l}\text { Bloqueos frecuentes y la palabra al decir no saldrá } \\
\text { por un momento, la lucha puede ser evidente }\end{array}$ \\
\hline $\begin{array}{l}\text { No muestran otros comportamientos proble- } \\
\text { máticos al hablar }\end{array}$ & $\begin{array}{l}\text { Conducta de escape (movimientos repentinos para } \\
\text { escapar de un movimiento de habla detenida) }\end{array}$ \\
\hline $\begin{array}{l}\text { No muestran reacciones negativas o frustra- } \\
\text { ción al hablar }\end{array}$ & $\begin{array}{l}\text { Conducta de evitación (puede ser cambiar palabras } \\
\text { o usar arrancadores) }\end{array}$ \\
\hline Revisiones de silabas & Palabras fragmentadas \\
\hline Interjecciones o muletillas & Circunloquios \\
\hline Vacilaciones en sonidos & Subidas de tono y volumen \\
\hline Los problemas duran de semanas a meses & Los problemas persisten más de 6 meses \\
\hline
\end{tabular}

\section{Tratamiento la tartamudez}

Cuando los niños presentan "disfluencias" o los padres piensan que están tartamudeando, ellos pueden implementar una serie de estrategias para estimular a los infantes a establecer una comunicación efectiva y sentirse felices de hacerlo entre estos podemos destacar:

- Orientarle al niño: hablar despacio, respirar antes de pronunciar o apoyarse sobre la palabra del interlocutor, pensar primero después decir las cosas.

- Realizar actividades para el trabajo con la respiración, tales como; Inflar globos, soplar velas (o cualquier otro objeto que sea de su interés) aumentando la distancia paulatinamente.

- Realizar lectura en alta voz con expresividad y entonación respetando los signos de puntación.

- Narrar de forma oral apoyándose en láminas de cuentos de su interés, facilitando así la fluidez y el desarrollo del vocabulario.

- Utilizar el juego como actividad fundamental y por medio de este realizar la labor correctiva.

- Eliminar métodos educativos incorrectos: evitar someterlo a situaciones que provoquen tensión tales como hablar ante personas que no desee, mostrar sus conocimientos, no hacer consciente al niño de su dificultad para realizar satisfactoriamente la comunicación oral.

- garantizar una dinámica familiar adecuada, que el infante se sienta aceptado, querido, escuchado, comprendido y rodearlo de vivencias positivas.

- Estimular el desarrollo del niño, tratando de evitar siempre en él la ansiedad motivada por la comunicación y garantizando un adecuado régimen del día e higiene física, mental y ambiental. 
- Realizar conteos o sonidos sostenidos en un tiempo determinado sin interrupción, dando a entender siempre que es un juego, teniendo en cuenta la edad del niño. Ejemplo 1, 2, 3,4.... Aaaaaa.

La realización de estas actividades y el tener en cuenta estos consejos, sin dudas facilitará la comunicación de los niños (33-36).

Si observamos que el problema de disfluencia persiste y se vuelve crónico, debemos acudir a un logopeda, el cual diagnosticara al infante dando un tratamiento más efectivo por medio de terapias. Actualmente, existen métodos de diagnóstico modernos que permiten diferenciar errores normales de la fluidez, de las expresiones indicadoras del inicio de una tartamudez; a partir de esto se pueden establecer distintos tipos de terapia o de intervención. Si es necesario intervenir directamente con el niño (directa) o hacerlo a través de sus progenitores (indirecta), forma que, habitualmente se utiliza en niños pequeños (36-39).

La terapia indirecta es aquella que no va dirigida a la disfluencia, al contrario, van administradas a la modificación del entorno del niño para que la alteración no se agrave. No se centra en el infante más bien en el ambiente en el que está presente. Por ello, lo que se pretende con este tipo de intervención es modificar y eliminar los estresores que puedan influir en la aparición y mantenimiento del trastorno. Dentro de esta clase encontramos la de grupo, asistencia de interacción madre/padre-hijo, prevención de la tartamudez temprana y cognitivo-conductual.

La Terapia directa está dirigida a la intervención de la disfluencia, se dan estrategias al niño para que pueda actuar frente al trastorno. Este tipo incluye la respiración regulada, el entrenamiento rítmico del habla, el programa global de la tartamudez y el Lidcombe que es un tratamiento conductual para niños

\section{Discusión y conclusiones}

A partir de las fuentes encontradas descubrimos que las disfluencias fisiológicas o tartamudez sigue siendo un tema muy investigado, por ello encontramos una gran variedad de artículos, aunque muchos de ellos van en direcciones opuestas. Sobre todo, cuando hablamos de su etiología, ya que aún no se conoce una causa exacta.

Las disfluencias fisiológicas en edad preescolar suelen aparecer entre los 3 y 5 años, pueden ser muy frecuentes en los niños que están desarrollando las habilidades de comunicación, aunque no se da en todos los casos. Por lo tanto, muy pocas veces suele volverse severo, lo que correspondería al $1 \%$ con datos epidemiológicos, diagnosticándose como una disfemia según el DSM-V, afectando en el habla del niño permanentemente si no hay una adecuada intervención (40).

Las disfluencias fisiológicas suelen durar un mínimo de 6 meses, es decir que el niño dejara de tartamudear por si solo sin ningún tipo de intervención.

Esta disfluencia que el niño presentara son: repeticiones de sílabas, palabras o frases, interjecciones, vacilaciones entre otras dificultades que no suelen ser muy severas. Su diagnóstico diferencial con la disfemia parte del número de fallas que el infante presenta al hablar, esto quiere decir que los errores serán menores en las disfluencias fisiológicas.

Si observamos dificultad al hablar en el niño debemos de tomar en cuenta ciertas 
pautas o estrategias que nos ayudaran a que ellos puedan no sentirse presionados entre estas esta:

- Escuchar con atención lo que el niño quiere decirte, responde al contenido y no a la forma como lo dice.

- Mantén el contacto visual natural cuando el niño esté hablando.

- No apresures al niño interrumpiéndole o terminando las palabras por él.

- No le ayudes completando lo que él quiere decirte, no permitan también que otros lo hagan.

- Cuando el niño que tartamudea hable fluidamente, no le digas frases "como lo hiciste bien" o "te felicito estás hablando mucho mejor", esto hace que se sienta evaluado cada vez que habla.

Es importante tomar en cuenta estos aspectos, así para no causar al niño o niña algún tipo de incomodidad o vergüenza $(1,41)$.

Al contrario, si hablamos de disfemia, persiste por más de 6 meses y no desaparece por sí sola, presenta con ella movimientos al hablar. Comportamientos como, conductas de escape o evitación al momento de expresarse, por otro lado, el número de errores en su habla será mucho mayor, presentando bloqueos, repeticiones, circunloquios y movimientos asicados. Por ello es un trastorno que si requiere de intervención logopédica para que estos niños puedan utilizar las estrategias dadas en su diario vivir ayudando a tener una comunicación eficaz.

\section{Conflictos de Intereses}

Los autores declaramos no tener conflictos de interés para el presente estudio.

\section{Referencias bibliográficas}

1. El Kadaoui Calvo M, Molina Gutiérrez MA, Gómez Andrés D. Enfoque y manejo de la tartamudez. Pediatría Aten Primaria. 2015; 17(65):49-51.

2. Jaramillo JH, González CJA. La tartamudez como un fenómeno prearticulatorio. Acta Neurol Colomb. 2008; 25(1):25-33.

3. Bermejo Quinche MT, Ambrosi Moreira JV. Evaluación e intervención logopédica de los trastornos del habla en los alumnos de la Escuela Dolores Josefina Torres, Cuenca, enero-julio 2012 [Internet] [Tesis de Pregrado]. [Ecuador]: Universidad de Cuenca; 2013 [citado 1 de septiembre de 2020]. Disponible en: http://dspace. ucuenca.edu.ec/handle/123456789/3795

4. El tartamudeo [Internet]. NIDCD. 2015 [citado 12 de septiembre de 2020]. Disponible en: https://www.nidcd.nih.gov/es/espanol/el-tartamudeo

5. Donaghy MA, Smith KA. Management options for pediatric patients who stutter: current challenges and future directions. Pediatr Health Med Ther. 2016; 7:71-7.

6. Kalinowski J, Saltuklaroglu T, Dayalu VN, Guntupalli V. Is it possible for speech therapy to improve upon natural recovery rates in children who stutter? Int J Lang Commun Disord. 2005;40(3):349-58.

7. Velázquez SC, Gil EM, Ortiz VV, Díaz DV. La Expresión Oral en el niño preescolar. XIKUA Bol Científico Esc Super Tlahuelilpan [Internet]. 2017 [citado 1 de septiem- 
bre de 2020];5(9). Disponible en: https://repository.uaeh.edu.mx/revistas/index. php/xikua/article/view/2240

8. Pedro Sánchez Escobedo. Psicología clínica - Pedro Sánchez Escobedo - Google Libros. Mexico. 2008.

9. Cervera JF, Ygual A. Intervención educativa en los niños tartamudos: retos técnicos y compromiso ético para los especialistas en audición y lenguaje. Edetania Estud Propues Educ. 2002;10(1):9-35.

10. Fernández-Zúñiga Marcos de León A, Gambra Moleres S. Evaluación, intervención y evolución en un caso de tartamudez temprana. Bol Aelfa. 2011;11(2):39-44.

11. Torres Ramos MJ, Gamarra Bezada IR. Nivel y tipo de tartamudez en los estudiantes del segundo grado de educación primaria de las instituciones educativas estatales de la zona urbana y urbano marginal de la provincia de Huarmey en el año 2011. [Internet]. [Peru]: Universidad Catolica los Angeles de Chimbote; 2011 [citado 1 de septiembre de 2020]. Disponible en: http://repositorio.uladech.edu.pe/ handle/123456789/58

12. Pazo Quintana T, Viduera Tamayo I, López Blanco N, Urrusuno Carvajal F, Llanes Álvarez MR. Valoración logofoniátrica de niñas y niños antes de iniciar el círculo infantil. Rev Cuba Pediatría. 2003; 75(3):10-4.

13. Pedraza PP, López TS. Desarrollo de la comunicación y del lenguaje: indicadores de preocupación. Rev Pediatr Aten Primaria. 2006;8(32):678-93.

14. Estepa EN. Disfemia: una revisión bibliográfica [Internet] [Tesis de Pregrado]. [Esapaña]: Universitat de les Illes Balears; 2012 [citado 1 de septiembre de 2020]. Disponible en: https://dspace.uib.es/xmlui/bitstream/handle/11201/212/Navarro_ Elisabeth.pdf?isAllowed=y\&sequence $=1$

15. Traverso N. Disfluencia y familia [Internet] [Tesis de Pregrado]. [Mar del PlataArgentina]: Universidad FASTA; 2015 [citado 1 de septiembre de 2020]. Disponible en: http:// redi.ufasta.edu.ar:8080/xmlui/bitstream/handle/123456789/1011/2015_F_007.pdf?sequence

16. Butcher JN, Mineka S, Hooley J. Psicología clínica. $12^{\circ}$. Madrid España: Pearson Educación; 2010.

17. Karina $\mathrm{BH}$. La incidencia de la tartamudez en el desarrollo del lenguaje de los niños y niñas de 5 a 6 años. Propuesta elaboración de un manual didáctico para docentes y representantes legales [Internet] [Tesis de Pregrado]. [Guayaquil Ecuador]: Universidad de Guayaquil; 2015 [citado 1 de septiembre de 2020]. Disponible en: http://repositorio.ug.edu.ec/bitstream/redug/12898/1/Beltr\%C3\%A1n\%2OHuayamabe $\% 2 \mathrm{C} \% 20$ Karina.pdf

18. Campos Fernandez E, Caño Bueno M, Carrion Espada D, Diaz Chacon TA, Gallardo Aragon C, Garceran Rodriguez B. Didáctica de los Trastornos del Ritmo en Primaria: La Disfemia. 1. Malaga España: Guillermo Castilla; 2008.

19. De Valladolid U, Página TS. Aceptando la tartamudez. Jorge Gutiérrez Ornia. 2015.

20. Brosch S, Winkler S. Diagnostics and therapy of stuttering children. Laryngorhinootologie. 2008;87(7):511-8; quiz 519-23. 
21. Jaramillo JH, González CJA. La tartamudez como un fenómeno prearticulatorio. Acta Neurol Colomb. 2009;25(1):25-33.

22. Cruz C, Amorim H, Beca G, Nunes R. Neurogenic stuttering: a review of the literature. Rev Neurol. 2018;66(2):59-64.

23. Marta Soler Roger. Disfemia o Tartamudez en la niñez | Gabinete Psicopedagógico | Sermesa [Internet]. 2007 [citado 13 de octubre de 2020]. Disponible en: https:// sermesa.es/2007/05/20/la-disfemia-o-tartamudez-en-la-ninez/

24. Barros PM de L, Oliveira PN de. Perfil dos pacientes atendidos no setor de fonoaudiologia de um serviço público de Recife - PE. Rev CEFAC. 2010;12(1):128-33.

25. Alvanta logopedia psicologia. Elementos para la detección de la Disfemia (Tartamudez) - Linares28 [Internet]. 2012 [citado 17 de octubre de 2020]. Disponible en: https://www.linares28.es/2012/06/03/elementos-para-la-deteccion-de-la-disfemia-tartamudez/

26. Sugathan N, Maruthy S. Predictive factors for persistence and recovery of stuttering in children: A systematic review. Int J Speech Lang Pathol. 2020;1-13.

27. Capote LLA. Métodos logopédicos y técnicas acupunturales. Microsistema auricular en el tratamiento de la tartamudez. Rev Conrado [Internet]. 2012 [citado 1 de septiembre de 2020];8(32). Disponible en: https://conrado.ucf.edu.cu/index.php/ conrado/article/view/42

28. Szkiełkowska A, Ratyńska J, Czyzewski A, Skarzyński H, Kurkowski M, Mularzuk $\mathrm{M}$, et al. [Plan of therapy and rehabilitation in stuttering patients]. Otolaryngol Pol Pol Otolaryngol. 2003;57(4):555-9.

29. Ogasawara M, Takeda M. [Stuttering/stammering]. Ryoikibetsu Shokogun Shirizu. 2003;(40):84-6.

30. Rodríguez Morejón A. Intervención temprana en tartamudez: criterios para tomar decisiones. Rev Logop Foniatría Audiol. 2000;20(3):136-50.

31. Ramírez LH. Tratamiento de la tartamudez por los procedimientos de auto-control. Rev Latinoam Psicol. 1975;7(3):421-34.

32. La tartamudez en los niños pequeños y en edad preescolar: ¿qué es lo típico y qué no lo es? [Internet]. HealthyChildren.org. [citado 8 de septiembre de 2020]. Disponible en: https://www.healthychildren.org/Spanish/ages-stages/toddler/Paginas/ Stuttering-in-Toddlers-Preschoolers.aspx

33. García Morales D, Morejón Barrueto Y. Consejos a la familia para evitar la aparición de la tartamudez. MediSur. 2015;13(5):575-7.

34. Asatiani NM, Kalacheva IO, Baranova MA. [Role of psychotherapy in the complex treatment of children who stutter]. Zhurnal Nevropatol Psikhiatrii Im SS Korsakova Mosc Russ 1952. 1981;81(10):1544-7.

35. Cave D. Assessment and treatment of stuttering in children. Dev Med Child Neurol. 1977;19(3):410-2.

36. Logan KJ, Caruso AJ. Parents as partners in the treatment of childhood stuttering. Semin Speech Lang. 1997;18(4):309-26. 
37. Franken MC. [Fifty years of treating stuttering in young children]. Ned Tijdschr Geneeskd. 2004;148(33):1622-6.

38. Koushik S, Shenker R, Onslow M. Follow-up of 6-10-year-old stuttering children after Lidcombe program treatment: a phase I trial. J Fluen Disord. 2009;34(4):27990.

39. Haapanen M-L. [Treatment of stuttering]. Duodecim Laaketieteellinen Aikakauskirja. 2009;125(19):2093-8.

40. Fundación Americana de la Tartamudez. Prevalencia e Incidencia | La Fundación Americana de la Tartamudez [Internet]. 2020 [citado 16 de septiembre de 2020]. Disponible en: https://www.tartamudez.org/prevalencia-e-incidencia

41. Murray FP. [Stuttering. Theory and treatment]. Acta Otorinolaryngol Iber Am. 1969;20(2):166-71. 